\title{
Uso de ácido ascórbico en pacientes con traumatismo craneoencefálico severo y su impacto en el tiempo de estancia en la Unidad de Cuidados Intensivos adultos
}

Ascorbic acid use in severe traumatic brain injury patients and its impact on the adult Intensive Care Unit stance Uso de ácido ascórbico em pacientes com traumatismo cranioencefálico grave e seu impacto no tempo de permanência na unidade de terapia intensiva para adultos

\section{Juan Diego de Jesús Macías Limón, ${ }^{\star}$ Alma Erika Hernández Plata, ${ }^{\star}$ Edgar Bravo Santibáñez, ${ }^{\star}$ Martha Alicia Hernández}

\section{RESUMEN}

Introducción: El traumatismo craneoencefálico (TCE) es la principal causa de muerte y discapacidad en adultos jóvenes alrededor del mundo. La lesión secundaria se desarrolla con liberación de radicales libres de oxígeno. Además de su potente acción como reductor de radicales libres, el ácido ascórbico estabiliza el endotelio y promueve la integridad de la barrera hematoencefálica (BHE). Objetivo: Determinar la utilidad del ácido ascórbico en pacientes con TCE severo y su impacto en el tiempo de estancia hospitalaria en la Unidad de Cuidados Intensivos adultos (UCl).

Material y métodos: Ensayo clínico aleatorizado, abierto en UCI del Centro Médico Nacional del Bajío UMAE No. 1, León, Guanajuato, México, de abril 2020 a julio 2020. Ingresaron 24 pacientes (12 grupo experimental y 12 controles) mediante muestreo probabilístico con TCE severo en mayores de 18 años. Administración de ácido ascórbico un gramo cada seis horas intravenoso. Se correlacionaron administración de ácido ascórbico con tiempo de estancia hospitalaria en $\mathrm{UCl}$ y hospitalización, mortalidad y escalas neurológicas. Análisis de resultados SPSS versión 21

Resultados: Se incluyeron 24 pacientes, medias de edad 26 y 49 años grupo experimental y control respectivamente $(p=0.004)$. Promedio de la Escala de Coma de Glasgow al momento del ingreso fue 7 y 6.7 puntos respectivamente $(p=0.378)$. Estancia en la UCI con 7 y 3.71 días $(p=0.001)$, estancia hospitalaria 12.5 y 5.85 días respectivamente $(p=0.001)$. Al egreso, la escala de Glasgow 3.50 y 3.57 puntos respectivamente $(p=0.630)$. Mortalidad a 28 días de 1 y 3 casos respectivamente $(p=0.59)$; el análisis de supervivencia reflejó mayor supervivencia para el grupo experimental $(p=0.010)$.

Conclusión: El uso de ácido ascórbico en TCE severo impactó de manera negativa en la estancia en $\mathrm{UCI}$, así como tiempo de hospitalización. El análisis de supervivencia mostró menor mortalidad a 28 días para el grupo experimental respecto al control.

Palabras clave: Traumatismo craneoencefálico, ácido ascórbico, estancia en Unidad de Cuidados Intensivos, mortalidad.

\section{ABSTRACT}

Introduction: Traumatic brain injury (TBI) is the leading cause of death and disability in young adults around the world. The secondary lesion develops with the release of oxygen free radicals. In addition to its powerful action as a free radical scavenger, ascorbic acid stabilizes the endothelium and promotes the integrity of the blood-brain barrier (BBB).

Objective: To determine the usefulness of ascorbic acid in patients with severe TBI and its impact on the length of hospital stay in the Adult Intensive Care Unit (ICU). Material and methods: Randomized clinical trial, open in the ICU of the Centro Médico Nacional del Bajío UMAE No. 1, León, Guanajuato, Mexico, from April 2020 to July 2020. 24 patients (12 experimental group and 12 controls) were admitted by probabilistic sampling with Severe TBI in people over 18 years of age. Administration of ascorbic acid one gram every six hours intravenously. Ascorbic acid administration was correlated with length of stay in ICU and hospitalization, mortality and neurological scales. Analysis of SPSS version 21 results.

Results: 24 patients were included, mean ages 26 and 49 years, experimental and control group respectively $(p=0.004)$. Average of the Glasgow Coma

\footnotetext{
* Unidad Médica de Alta Especialidad, Centro Médico Nacional Bajío No. 1. Instituto Mexicano del Seguro Social.

Recibido: 16/10/2020. Aceptado: 09/11/2020

Citar como: Macías LJDJ, Hernández PAE, Bravo SE, Hernández MA. Uso de ácido ascórbico en pacientes con traumatismo craneoencefálico severo y su impacto en el tiempo de estancia en la Unidad de Cuidados Intensivos adultos. Med Crit. 2021;35(3):136-143. https://dx.doi.org/10.35366/100002

www.medigraphic.com/medicinacritica
}

Scale at the time of admission was 7 and 6.7 points respectively ( $p=0.378$ ). Stay in the ICU with 7 and 3.71 days $(p=0.001)$, hospital stay 12.5 and 5.85 days respectively $(p=0.001)$. At discharge, the Glasgow scale resulted in 3.50 and 3.57 points respectively $(p=0.630)$. 28-day mortality of 1 and 3 cases respectively $(p=0.59)$; the survival analysis reflected higher survival for the experimental group $(p=0.010)$.

Conclusion: The use of ascorbic acid in severe TBI had a negative impact on the ICU stay, as well as the length of hospitalization. Survival analysis showed lower mortality at 28 days for the experimental group compared to the control. Keywords: Head trauma, ascorbic acid, stay in Intensive Care Unit, mortality.

\section{RESUMO}

Introdução: O traumatismo cerebral (TCE) é a principal causa de morte e incapacidade em jovens adultos em todo o mundo. A lesão secundária se desenvolve com a liberação de radicais livres de oxigênio. Além de sua poderosa ação como redutor de radicais livres, o ácido ascórbico estabiliza o endotélio e promove a integridade da barreira hematoencefálica (BHE).

Objetivo: Determinar a utilidade do ácido ascórbico em doentes com TCE grave e o seu impacto no tempo de hospitalização na Unidade de Cuidados Intensivos (UTI) de adultos.

Material e métodos: Ensaio clínico aleatório, aberto na UTI do Centro Médico Nacional del Bajío UMAE No. 1, León, Guanajuato, México, de Abril de 2020 a Julho de 2020. Vinte e quatro pacientes (12 grupos experimentais e 12 controlos) foram admitidos por amostragem probabilística com TCE grave em pacientes com mais de 18 anos de idade. Administração de ácido ascórbico uma grama a cada seis horas por via intravenosa. Correlacionou-se a administração do ácido ascórbico com o tempo de estadia hospitalar na UTI e a hospitalização, mortalidade e escalas neurológicas. Análise de resultados SPSS versão 21.

Resultados: 24 pacientes foram incluídos, idade média de 26 e 49 anos, respectivamente, grupo experimental e grupo de controle $(p=0.004)$. A Escala Média de Coma de Glasgow na admissão foi de 7 e 6.7 pontos respectivamente $(p=0.378)$. A estadia na UCI foi de 7 e 3.71 dias $(p=0.001)$, a estadia hospitalar foi de 12.5 e 5.85 dias, respectivamente $(p=0.001)$. Na alta, a escala Glasgow pontua 3.50 e 3.57, respectivamente $(p=0.630)$. Mortalidade a 28 dias de 1 e 3 casos respectivamente ( $p=0.59$ ); a análise de sobrevivência reflectiu uma maior sobrevivência para o grupo experimental $(p=0.010)$.

Conclusão: $O$ uso de ácido ascórbico em TCE grave teve impacto negativo na permanência na UTI, bem como no tempo de internação. A análise de sobrevivência mostrou menor mortalidade em 28 dias para o grupo experimental em comparação com o controle.

Palavras-chave: Traumatismo craniano, ácido ascórbico, permanência na Unidade de Terapia Intensiva, mortalidade.

\section{INTRODUCCIÓN}

El traumatismo craneoencefálico (TCE), según la CDC, es una disrupción de la función normal del cerebro que puede ser causada por una contusión o sacudida a la cabeza, o una lesión penetrante a la cabeza. ${ }^{1}$ También puede ser definido como «cualquier lesión estructural o funcional del cráneo y/o su contenido secundario a un intercambio brusco de energía mecánica».2,3

EI TCE ha tenido presencia ubicua en la historia del ser humano, ya que su existencia formaba parte del día a día; sin embargo, en la actualidad, su prevalencia alarmante ha sido motivo de estudio y de múltiples es- 
trategias para su prevención y tratamiento porque continúa siendo la principal causa de muerte y discapacidad en adultos jóvenes alrededor del mundo. En la historia más reciente sobre esta entidad, el desarrollo de tecnología como lo es la tomografía axial computada (TAC), ha sido fundamental para su estudio y así dar lugar a múltiples clasificaciones. Se han formulado escalas clínicas como la Escala de Coma de Glasgow (ECG) y, lo más reciente, búsqueda de métodos confiables para la monitorización de la presión intracraneal, paralelamente con el concepto de «prevención de lesión cerebral secundaria», las cuales han ayudado al diagnóstico, pronóstico y mejora de resultados posteriores al TCE. Aunque es evidente que por la fisiopatología y mecanismos que intervienen en su evolución, se siguen generando controversias sobre el manejo en la Unidad de Cuidados Intensivos (UCI). ${ }^{4}$

Como es sabido, el TCE es uno de los problemas de salud pública más importantes en Estados Unidos de América y en el mundo entero, resultando en 2.8 millones de visitas al departamento de emergencias y 56,000 muertes anuales. Por lo que representa la principal causa de discapacidad en niños y adultos entre uno y 44 años. 5,6

En nuestro país, según el SINAVE (Sistema Nacional de Vigilancia Epidemiológica), el TCE ocupa la tercera causa de muerte, precedido por causas cardiovasculares y cáncer. Fue la causa de 35,567 defunciones en el año 2008 , lo que conlleva a una mortalidad de $38.8 \%$ por cada 100,000 habitantes. ${ }^{7}$ Respecto a la relación hombre:mujer, es mayor el del varón 3:1, siendo mayormente afectada la población de 15 a 45 años. Setenta y cinco por ciento de las causas de TCE son los accidentes de tráfico, afectando a menores de 25 años, motociclistas y personas que manejan en estado de ebriedad. Veinte por ciento se relaciona con caídas, siendo los niños menores de cinco años y adultos mayores a 65 años los más afectados; por último, lesiones deportivas y agresiones en $5 \%$. 2,7

Existen varias clasificaciones para el TCE, sin embargo, la ECG ha demostrado relación inversamente proporcional con la mortalidad y resultados, llegando a clasificar al TCE en leve, moderado y severo, con puntuaciones de 15-14, 13-9 y $\leq 8$ puntos respectivamente, tomando en cuenta variables como la respuesta ocular, respuesta motora y verbal otorgando puntuaciones de $1-4,1-6,1-5$ puntos, con una sensibilidad de $84.2 \%$ y especificidad de $88.6 \% .^{3,4,8-13}$

Existen otras escalas de clasificación y pronóstico basadas en los hallazgos tomográficos, por lo que la TAC de cráneo es el estudio de imagen inicial utilizado en pacientes con sospecha de TCE. De igual forma, es el único método aceptado por la administración de alimentos y fármacos de los Estados Unidos de América (FDA), además es utilizada por su sensibilidad para identificar hemorragia intracraneal que amenaza la vida, contusión, efecto de masa y fracturas, tiene amplia disponibilidad y bajo costo, la misma añade información que dirige al triaje médico y la toma de decisiones quirúrgicas, así como pronóstico de recuperación. La clasificación de Marshall modificada se enfoca en el estado de las cisternas de la base, el grado de desviación de la línea media, presencia de lesión de masa y criterios para el drenaje quirúrgico con una sensibilidad de $52.4 \%$ y una especificidad de $93.7 \%$, clasificándolo en 4 categorías: 1) no existe condición patológica visible; no es visible compresión intracraneal; 2) existe condición patológica intracraneal, las cisternas están presentes pero existe un desplazamiento de la línea media de 1-5 mm; 3) existe condición patológica intracraneal, las cisternas son comprimidas o ausentes, con desviación de la línea media de 0-5 mm; y 4) existe condición patológica intracraneal, desviación de la línea media $>5$ $\mathrm{mm}^{4,11,12,14}$

Para el entendimiento de la fisiopatología del TCE, clásicamente es dividido en dos distintas fases, lesión primaria seguida de una lesión secundaria retardada. La lesión primaria involucra fuerzas físicas externas aplicadas a la cabeza produciendo fracturas del cráneo, hematomas y deformación y destrucción del tejido cerebral, incluyendo contusiones y lesión axonal, en esta fase no podemos intervenir ni incidir. La lesión secundaria se desarrolla a través del tiempo con la activación de múltiples vías moleculares y celulares; el impacto de la lesión cerebral secundaria causada por la disautorregulación de los vasos cerebrales y disrupción a la barrera hematoencefálica (BHE) podrían incrementar las complicaciones, llevando a edema cerebral, aumento en la presión intracraneal, y finalmente disminución en la presión de perfusión cerebral. Sin embargo, estos procesos de lesión cerebral incorporan muchos factores clínicos: despolarización y afección de la homestasis iónica, liberación de neurotransmisores (glutamato por exocitosis), disfunción mitocondrial, apoptosis neuronal, degradación lipídica, iniciación de respuestas inflamatorias e inmunes, así como un aumento en los radicales libres de oxígeno, lo que conlleva a disfunción celular e incluso a muerte celular. ${ }^{12,15}$

Los radicales libres de oxígeno causan daño a través de la peroxidación lipídica de los ácidos grasos en las células y en las membranas mitocondriales, con sus propiedades antioxidantes potentes, altas dosis de ácido ascórbico podrían mitigar esta cascada. ${ }^{6,16}$

Los enfermos críticos se caracterizan por una depleción de las reservas de vitamina $\mathrm{C}$, por lo que la administración de antioxidantes, incluyendo ácido ascórbico, que en estados de enfermedad crítica han reemergido como un área prometedora de estudio debido a su efectividad significativa en cohortes pequeñas en pacientes con choque séptico, y apoyado en la investigación en trauma, 
alivio de heridas, hematología; estas lesiones resultan en una desregulación del sistema inmune, altos niveles de radicales libres de oxígeno y fuga capilar debido a daño endotelial e incremento en la permeabilidad. ${ }^{6,17,18}$

\section{Sistemas de regulación cerebral}

El cerebro normal cuenta con algunos mecanismos para regular la presión y el volumen. El propósito de estos mecanismos es mantener un flujo sanguíneo cerebral continuo, así como una adecuada entrada de oxígeno a pesar de los cambios en presión arterial sistémica y los requerimientos metabólicos cerebrales. El mecanismo clave es el cambio en la resistencia cerebrovascular a través de la vasoconstricción y dilatación que se ajustan usando diferentes mediadores. La reactividad cerebral a la presión es uno de los sistemas críticos en la autorregulación y permite al músculo liso responder a cambios en la presión arterial sistémica, con ello mantiene el flujo sanguíneo cerebral (FSC) constante. Los vasos pequeños en el cerebro reaccionan a la presión hidrostática y regulan el tono vascular para mantener un FSC constante con presión arterial media entre 60 y $160 \mathrm{mmHg}$. Cuando los mecanismos de autorregulación fracasan y la BHE es afectada, el FSC se vuelve dependiente de la presión arterial sistémica, resultando en una condición crítica para el cerebro dañado, con lo que cambios accidentales en la presión arterial sistémica pueden llevar a condiciones dañinas e irreversibles como hipoperfusión (isquemia) o hiperperfusión (hiperemia). Estos pueden llevar a un incremento en la presión intracraneal (PIC) y a resultados catastróficos. ${ }^{15}$

Posterior a un TCE, el primer contribuyente para el fallo de la BHE es la disrupción mecánica inmediata posterior a la lesión primaria. El edema contusional es generado desde un potencial osmótico a través del tejido necrótico central (alta osmolaridad) y cerebro subyacente. Asimismo, además de esta causa inmediata, existen otros factores como lo son cascadas de segundos mensajeros, incluyendo citocinas proinflamatorias, lo que lleva a neuroinflamación, factores angiogénicos, degradación de uniones fuertes y moléculas de adhesión, factores que promueven la extravasación proteica y reacomodos citoesqueléticos. La fuga capilar proteínica al espacio intersticial puede incrementar la presión oncótica y ocluir vasos pequeños, causando hipoperfusión/isquemia que puede exacerbar la falla iónica. ${ }^{12,19}$

\section{Monitoreo y manejo del TCE en UCI}

Los pacientes con TCE severo son tratados en la $\mathrm{UCl}$, con un manejo especializado neurointensivo, combinado con estrategias utilizadas en la $\mathrm{UCl}$ de manera general como lo es alimentación enteral temprana, control y tratamiento de infecciones, normalización del inter- cambio respiratorio, fisioterapia, ventilación artificial y optimización de fluidos para mantener presión arterial y perfusión orgánica, así como el drenaje quirúrgico del hematoma. Este manejo se basa en prevenir insultos secundarios y a mantener la homeostasis cerebral. ${ }^{12}$

La lesión secundaria posterior al traumatismo es la etapa en la cual se deben enfocar todos los esfuerzos terapéuticos, ya que es en ésta donde se involucran amenazas sistémicas (hipoxia, hipercapnia, hipotensión arterial, hiponatremia, pirexia) y amenazas intracraneales (hematomas en expansión o contusiones e incrementos en la PIC). ${ }^{12}$

En la actualidad, las estrategias de tratamiento para mitigar la lesión cerebral secundaria están limitadas a la optimización de la condición clínica general, incluyendo el mantenimiento de las presiones de perfusión cerebral adecuadas, manejo de la hipertensión intracraneal y prevención de la hipotensión, hipoxia y convulsiones. No se ha demostrado un tratamiento efectivo para permitir la neuroprotección en la fase aguda o para promover la neurorregeneración en la ventana terapéutica retardada posterior al TCE. 6,20

El término neuroprotección se define como la estrategia que se ocupa de la prevención de la muerte neuronal, al inhibir uno o más de los pasos fisiopatológicos en los procesos que siguen a lesión del sistema nervioso o isquemia debido a oclusión de alguna arteria, o a hipoxia debido a cualquier causa; incluye intervenciones que enlentecen o frenan la degeneración neuronal.

La neuroprotección es importante en lesiones del sistema nervioso central (SNC). El daño inmediato desde la lesión podría no ser reversible, pero la progresión de la cadena de eventos que agravan el daño cerebral puede ser prevenida por una estrategia efectiva de neuroprotección. ${ }^{20}$

Una vez que la injuria ha finalizado, existen escalas que nos determinan pronósticos tanto funcionales como de recuperación y de secuelas, entre ellas destacan la escala de Rankin modificada y la escala de recuperación de Glasgow (Glasgow Outcome Scale); la escala de Rankin modificada fue instaurada por el Dr. John Rankin en 1957, siendo utilizada como escala en ictus isquémicos; sin embargo, se modificó en 1988 por van Swieten con siete valores: 0 asintomáticos, 1 sin discapacidad significativa, 2 discapacidad leve, 3 discapacidad moderada, 4 discapacidad moderadamente severa, 5 discapacidad severa y 6 muerte. ${ }^{21}$

Asimismo, la escala de recuperación de Glasgow que fuera publicada en 1975 por Byan Jennet y Michael Bond, describen la recuperación que presentaría un paciente que ha sido sometido a un trauma craneoencefálico otorgando cinco niveles, mismos que se describen a continuación: 1 muerte, 2 estado neurovegetativo, 3 discapacidad severa, 4 discapacidad moderada, 5 adecuada recuperación. ${ }^{22}$ 


\section{Vitamina C en TCE}

Dentro de las medidas de neuroprotección, se describe el uso del ácido ascórbico, también denominado vitamina $C$, que es una vitamina hidrosoluble que se encuentra naturalmente presente en algunos alimentos, así como en suplementos dietéticos. Los humanos, así como otros animales, son incapaces de sintetizar ácido ascórbico de manera endógena, por lo que es un componente esencial de la dieta. La ingesta diaria recomendada en mujeres es de $75 \mathrm{mg}$ y en hombres es de 90 mg. Suplementar ácido ascórbico en la dieta a niveles superiores se sugiere para proveer de protección antioxidante contra el estrés oxidativo, que es característico de la lesión secundaria, ${ }^{23}$ funciona como un agente reductor de radicales libres, cofactor en numerosas reacciones biosintéticas. Se ha descrito que la ingesta de dosis mayores es requerida para alcanzar mayores niveles de reducción de radicales libres, principalmente en estados de lesión aguda o enfermedad crítica. ${ }^{6}$

Las concentraciones normales en personas sanas de ácido ascórbico varían entre $21 \mu \mathrm{mol} / \mathrm{L}(0.37 \mathrm{mg} / \mathrm{dL})$ a $100 \mu \mathrm{mol} / \mathrm{L}(1.76 \mathrm{mg} / \mathrm{dL})$, la administración de ácido ascórbico de manera intravenosa resultó en elevaciones plasmáticas de $885 \pm 201.2 \mu \mathrm{mol} / \mathrm{L}$. En pacientes críticamente enfermos la administración de 2,000 mg de ácido ascórbico por día IV restauró los niveles plasmáticos, pero sólo una dosis de 10,000 mg elevó las concentraciones por encima de $1,000 \mu \mathrm{mol} / \mathrm{L}$, que es la concentración óptima para los efectos de reducción de radicales libres. En sepsis, Fowler y colaboradores, demostraron que la administración de $200 \mathrm{mg} / \mathrm{kg}$ por día (comparado con $50 \mathrm{mg} / \mathrm{kg} / \mathrm{día}$ ) en cuatro bolos, de manera efectiva y consistente, elevó la concentración por encima de $1,000 \mu \mathrm{mol} / \mathrm{L}$ dentro de las primeras 24 horas de la primer dosis; por lo tanto, un régimen de tratamiento de $50 \mathrm{mg} / \mathrm{kg}$ IV dado cada seis horas (un total de $200 \mathrm{mg} / \mathrm{kg} / \mathrm{día}$ ) resulta en niveles elevados de reducción de radicales libres en pacientes críticamente enfermos. ${ }^{24}$

En su forma oxidada, el ácido ascórbico es activo y preferentemente transportado al SNC, cruzando la BHE por transporte facilitado. ${ }^{25} \mathrm{La}$ administración de ácido ascórbico tiene pocos efectos adversos relevantes en el ámbito clínico. Como compuesto altamente hidrosoluble, es excretado por los riñones y orina, limitando el potencial de toxicidad sistémica, por lo tanto, toxicidad renal directa y formación de litos de oxalato son las complicaciones más comunes tras la administración de ácido ascórbico en altas dosis. ${ }^{26}$

Los pacientes críticamente enfermos toleraron la administración de 4,500 mg IV durante 28 días, e incluso dosis mayores se han encontrado en ensayos de sepsis y cáncer de fase 1, lo que demuestra que dosis elevadas son seguras y con bajo riesgo de toxicidad. 6,27
Las dosis que se han evaluado varían desde $15 \mathrm{mg} /$ $\mathrm{kg} / \mathrm{h}$ en estudios de animales y hasta $66 \mathrm{mg} / \mathrm{kg} / \mathrm{h}$ en ensayos clínicos. $6,16,28$

Los mecanismos de defensa contra el daño celular secundario a estrés oxidativo se encuentran dañados en enfermedad aguda, debido a la depleción de los reductores de radicales libres como lo es el ácido ascórbico. ${ }^{17,29}$ En un estudio de cohorte pequeño de pacientes con TCE e ictus hemorrágico, las concentraciones plasmáticas de ácido ascórbico se encontraron bajas e inversamente correlacionadas con la severidad del daño, se encontró un nivel plasmático significativamente inferior $(29 \pm 8 \mu \mathrm{mol} / \mathrm{L})$ que los controles saludables (52 $\pm 8 \mu \mathrm{mol} / L$ ), y niveles plasmáticos de ácido ascórbico inversamente correlacionados con el tamaño de la hemorragia. ${ }^{16}$ En adición a su potente acción como reductor de radicales libres, el ácido ascórbico puede estabilizar el endotelio y promover la integridad de la BHE después del impacto mecánico del TCE, isquemia, reperfusión y el desequilibrio entre disminución de factores protectores, como reductores de radicales libres y factor 2 relacionado NF-E2, y un aumento en los factores de daño, como la metaloproteinasa (MMP)-9, que llevan a la disrupción de las proteínas de uniones fuertes transmembrana en la lámina basal de la BHE.6,30

Los datos clínicos del uso terapéutico de antioxidantes a la fecha han sido inconclusos o decepcionantes. Existe un ensayo clínico controlado sobre el uso de ácido ascórbico en TCE, en el cual se utilizó una dosis elevada (10,000 mg en los días uno y cuatro; $4,000 \mathrm{mg}$ en los días cinco a siete) IV en 23 pacientes, lo que resultó en disminución del edema perilesional en la imagen TC ( $p=0.01$ ), pero sin mejoría en los resultados sobre la mortalidad. 6,31

La administración de ácido ascórbico a pacientes con TCE severo, a través de sus propiedades de reducción de radicales libres de oxígeno mismos que dañan la célula de manera directa, podría disminuir el tiempo de estancia hospitalaria en la Unidad de Cuidados Intensivos y, de esta manera, optimizar las medidas de neuroprotección.

\section{Aspectos de bioseguridad}

El ácido ascórbico se considera un suplemento alimenticio, puede presentarse urticaria y litiasis renal, en cuyo caso, se notificaría mediante la hoja de evento adverso de manera inmediata al comité de ética, el hospital cuenta con el equipamiento y personal capacitado para resolverlo.

\section{MATERIAL Y MÉTODOS}

Se realizó un ensayo clínico aleatorizado, abierto en la Unidad de Cuidados Intensivos del Centro Médico Na- 
cional del Bajío UMAE No. 1, León Guanajuato, México, de abril 2020 a julio 2020. Se ingresaron 24 pacientes (12 grupo experimental y 12 grupo control) mediante muestreo probabilístico de casos con TCE severo evaluados por Escala de Coma de Glasgow, en mayores de 18 años, ingresados en las primeras 24 horas del traumatismo a la Unidad de Cuidados Intensivos, se incluyeron ambos sexos, con previa aceptación al estudio por parte de los familiares para la administración de ácido ascórbico. Se excluyeron a aquellos pacientes que tuvieran antecedentes de toma de suplementos vitamínicos en los últimos seis meses previos al trauma, alergias conocidas a ácido ascórbico, así como aquéIlos que tuvieron TCE leve o moderado, epilepsia y enfermedad renal crónica. Se analizaron las características demográficas entre ambos grupos para conocer la distribución de variables y diferencias de ambos grupos mediante análisis de t de Student. Se inició la evaluación de los pacientes desde el área de urgencias y se realizó la aleatorización mediante uso de tómbola con fichas rojas y negras, las rojas fueron para los del grupo experimental y negras para el grupo control. Una vez incluidos en los grupos, se inició la administración de ácido ascórbico a dosis de un gramo cada seis horas intravenoso, (solución inyectable una ámpula de $10 \mathrm{~mL}$ conteniendo 1,000 mg de ácido ascórbico) que se administró en bolo por parte de personal de enfermería desde la llegada del paciente al Servicio de Urgencias y durante la estancia en la Unidad de Cuidados Intensivos, observando intencionadamente posibles reacciones secundarias al medicamento. Se realizaron tomografías computadas de cráneo para ambos grupos durante la llegada a urgencias, se midieron diferentes parámetros para integrar la escala de Rotterdam. Asimismo, se realizaron análisis de comparación entre ambos grupos de estudio para el objetivo primario, que fue determinar el impacto sobre los días de estancia hospitalaria tanto en UCI como hospitalización. Se determinó mortalidad a 28 días, escalas neurológicas al ingreso y al egreso hospitalario. Se analizaron los resultados por medio del programa estadístico SPSS versión 21.

\section{RESULTADOS}

Se incluyeron 24 pacientes que ingresaron al Servicio de Urgencias con diagnóstico de TCE severo, realizándose al momento del ingreso una aleatorización mediante tómbola para la integración del grupo experimental $(n=12)$ y el grupo control $(n=12)$.

Para el análisis final de la totalidad de los pacientes incluidos, se encontró una mediana (riq) de edad de 31 años (24-48.9 años); edad mínima 18 años y máxima 73 años; $12.5 \%(n=3)$ fueron mujeres, y $87 \%(n=21)$ fueron hombres; de los datos antropométricos, una mediana de peso de $75 \mathrm{~kg}(69.2-77.1)$ rango $50-110 \mathrm{~kg}$; talla media de $1.70 \mathrm{~m}( \pm 0.045 \mathrm{~m}$, rango $1.62-1.80 \mathrm{~m}), \mathrm{y}$ un IMC con una mediana de $26.07 \mathrm{~kg} / \mathrm{m}^{2}(24.2-26.5 \mathrm{~kg})$. Sólo dos pacientes presentaron comorbilidades (uno con DM2 y uno con HAS); al ingreso se presentó una mediana de escala de coma de Glasgow de 7 puntos (6.7-7, rango 3-8 puntos), todos los pacientes fueron clasificados como traumatismo craneoencefálico seve-

Tabla 1: Comparación de la población de acuerdo con la administración de ácido ascórbico y controles.

\begin{tabular}{|c|c|c|c|}
\hline Variable & $\begin{array}{l}\text { Grupo experimental (ácido ascórbico) } \\
\qquad(n=12)\end{array}$ & $\begin{array}{l}\text { Grupo control } \\
\qquad(n=12)\end{array}$ & $\mathrm{p}$ \\
\hline Género, n (\%) & & & $0.217^{\star}$ \\
\hline Masculino & $9(75)$ & $12(100)$ & \\
\hline Edad (años) & $26.75(21-29)$ & $49.28(48.3-58.5)$ & $0.004^{\ddagger}$ \\
\hline Peso (kg) & $77.1(71.2-79.5)$ & $75.42(67.5-75-8)$ & $0.028^{\ddagger}$ \\
\hline Talla (m) & $1.72(0.56)$ & $1.69(0.025)$ & $0.045^{\S}$ \\
\hline Escala Rotterdam & $3(2.8-3)$ & $3.71(3-3.92)$ & $0.007^{\ddagger}$ \\
\hline Escala APACHE ॥ & $19(16-21)$ & $13.6(12.6-17.6)$ & $0.052^{\ddagger}$ \\
\hline Escala SOFA & $11(7.25-12.46)$ & $7(4.5-8.75)$ & $0.032^{\ddagger}$ \\
\hline \multicolumn{4}{|l|}{ Datos tomográficos, $\mathrm{n}(\%)$} \\
\hline Desviación de la línea media & $9(75)$ & $9(75)$ & $0.999^{9}$ \\
\hline Uso terapia hiperosmolar, n (\%) & $11(91.7)$ & $10(83.3)$ & $0.999^{9}$ \\
\hline Glasgow Outcome & $3.50(3.13-4)$ & $3.57(3.14-4)$ & $0.630^{\ddagger}$ \\
\hline \multicolumn{4}{|l|}{ Desenlace, n (\%) } \\
\hline Defunción & $1(8.3)$ & $3(25)$ & $0.590^{*}$ \\
\hline
\end{tabular}

\footnotetext{
${ }^{*}=$ Exacta de Fisher. ${ }^{\ddagger}=\mathrm{U}$ de Mann Withney. ${ }^{\S}=\mathrm{T}$ de student. ${ }^{~}{ }^{\natural}=$ Chi cuadrada.
} 
Tabla 2: Análisis de riesgo del uso de ácido ascórbico con las variables: defunción, tratamiento quirúrgico y uso de terapia hiperosmolar.

\begin{tabular}{|c|c|c|c|}
\hline Variable & $n(\%)$ & Riesgo & IC al $95 \%$ \\
\hline Defunción ( $N=4)$ & & $\mathrm{HR}$ & \\
\hline & $1(25.0)$ & 0.128 & $0.039-0.422$ \\
\hline $\begin{array}{l}\text { Tratamiento quirúrgico } \\
(\mathrm{N}=13)\end{array}$ & & $\mathrm{RR}$ & \\
\hline \multirow{3}{*}{$\begin{array}{l}\text { Uso de terapia } \\
\text { hiperosmolar }(\mathrm{N}=21)\end{array}$} & $6(46.2)$ & 0.857 & $0.409-1.79$ \\
\hline & & $\mathrm{RR}$ & \\
\hline & $11(52.4)$ & 1.1 & $0.811-1.493$ \\
\hline
\end{tabular}

$\mathrm{HR}=$ hazard ratio; $\mathrm{RR}=$ riesgo relativo; $\mathrm{IC}$ al 95\% = intervalo de confianza al $95 \%$.

ro (TCE severo). Se realizó un análisis imagenológico previo al ingreso a la UCI (TAC de cráneo fase simple); $100 \%$ de los pacientes presentaron edema cerebral, $75 \%(n=18)$ desviación de la línea media, y se calculó la escala de Rotterdam con una mediana de tres puntos (3-3.7 puntos).

Una vez definidos ambos grupos, se observaron medias de edad de 26 años para grupo experimental y 49 años para grupo control $(p=0.004)$. El promedio de la Escala de Coma de Glasgow para el grupo experimental al momento del ingreso al estudio fue de 7 puntos y para el grupo control de $6.7(p=0.378)$. La escala de gravedad de APACHE II fue de 19 puntos para el grupo experimental y 13.6 para grupo control (0.052) y SOFA al ingreso de 11 puntos grupo experimental y 7 puntos grupo control $(p=0.032)$. Con respecto a los hallazgos tomográficos iniciales, no se observaron diferencias en ambos grupos en cuanto a desviación de línea media $(p=0.99)$ y el edema cerebral $(p=0.99)$; sin embargo, ya integrada la escala de Rotterdam se observó de 3 y 3.71 puntos para grupo experimental y control respectivamente $(p=0.007)$ (Tabla 1).

El uso de medidas antiedema cerebral mediante terapia hiperosmolar se empleó en 11 pacientes del grupo experimental y 10 del grupo control $(p=0.99)$ (Tabla 2).

En relación al punto primario del estudio, se observó una diferencia estadísticamente significativa en los días de estancia en la UCI con siete días para el grupo experimental y 3.71 días para el grupo control $(p=0.001)$ (Figura 1), así como para los días de estancia hospitalaria con 12.5 y 5.85 días respectivamente $(p=0.001)$.

Al egreso hospitalario, ambos grupos no mostraron diferencia en la escala de Glasgow outcome siendo de 3.50 puntos y 3.57 respectivamente $(p=0.630)$. Por último, se observó una mortalidad a los 28 días de un paciente para el grupo experimental y tres pacientes para el grupo control $(p=0.59)$; sin embargo, mediante análisis de supervivencia con un modelo de regresión de Cox, se obtuvo un HR de 0.128 (IC 95\% 0.039-0.422), con significancia estadística, además se realizó un aná- lisis por medio de curva de Kaplan-Meier con presencia de mayor supervivencia para el grupo experimental ( $p$ $=0.010$ ) (Figura 2).

\section{DISCUSIÓN}

EI TCE es un problema de salud pública a nivel mundial, siendo responsable de una alta tasa de mortalidad y causa de discapacidad en personas en edad productiva. Ahí radica la importancia de un manejo estandarizado y oportuno, para intentar disminuir la estancia hospitalaria, las complicaciones y la mortalidad, de igual forma, mejorar el pronóstico vital y funcional en estos pacientes. Desarrollar una serie de maniobras y tratamientos novedosos podría ayudar a lograr estos objetivos.

En el presente estudio, investigamos si el uso de un suplemento alimenticio como el ácido ascórbico en altas dosis tendría un impacto sobre el tiempo de estancia de los pacientes con TCE en la UCl así como en hospitalización, como lo sugiere Leichtle y colaboradores, al disminuir el edema cerebral por los mecanismos de barrido de los radicales libres de oxígeno, de igual forma Razmon y su equipo reportan que tras una lesión cerebral severa los niveles séricos de ácido ascórbico disminuyen, por lo que la sustitución en altas cantidades de forma intravenosa podría mejorar estos niveles y disminuir el nivel de edema cerebral $(p=0.01)$.

No se ha realizado algún estudio que reporte el impacto que tiene la administración de ácido ascórbico en pacientes con TCE severo sobre los días de estancia intrahospitalaria en la $\mathrm{UCl}$, simplemente lo reportan como tiempo hospitalario, mismo que Razmon y su grupo reportan como un mayor tiempo de estancia en el grupo

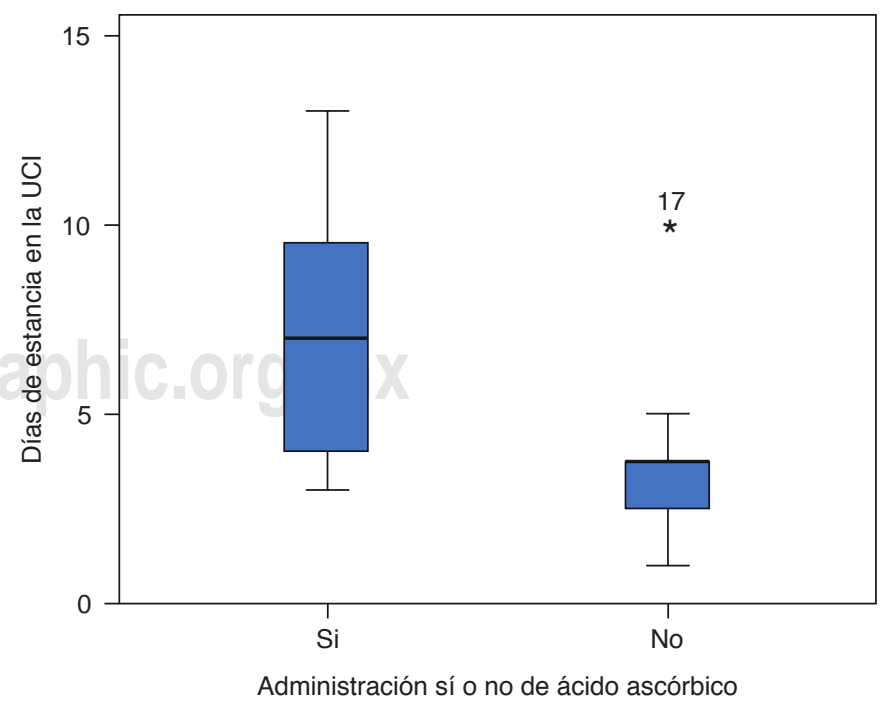

Figura 1: Comparación de medias del tiempo de estancia en la unidad de cuidados intensivos en los grupos de administración o no de ácido ascórbico. 


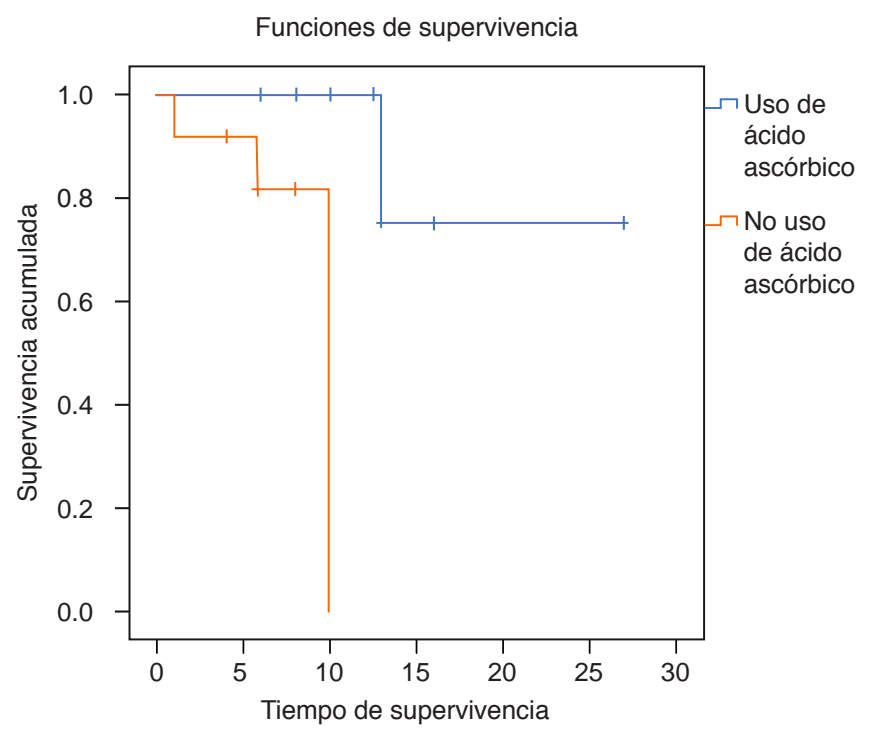

Figura 2. Curva de Kaplan-Meier para determinar supervivencia entre grupos de estudio. Con una prueba estadística de Mantel Cox de 6.645, con 1 grado de libertad, dando una $p=0.10$

de control respecto al de intervención $(p=0.08)$, lo cual coincide con lo obtenido en nuestro estudio, al presentar los pacientes del grupo de intervención una mayor estancia intrahospitalaria y en la $\mathrm{UCl}(p=0.001)$; en el caso particular de nuestro estudio, tal vez lo podríamos relacionar a que en la aleatorización de los pacientes se observaron puntajes más elevados en escalas de gravedad (APACHE II y SOFA), lo que pudiera justificar la estancia prolongada, por lo que creemos que para confirmar dicha premisa es necesaria la realización de estudios con mayor cantidad de pacientes, y cuyos grupos de estudio tengan similares características clínicas a fin de aclarar esta relación.

De la misma manera, en nuestro estudio se cuantificó la escala Glasgow outcome al egresar del hospital, observando que no hubo cambios en el pronóstico funcional de estos pacientes, tal y como lo sugiere Razmon y colaboradores, concluyendo que no existe al momento evidencia que sugiera favorecer o evitar el uso de ácido ascórbico en TCE severo, ya que los valores en ambos grupos de la escala de Glasgow outcome fueron similares $(p=0.04)$.

La necesidad de terapia hiperosmolar debido a edema cerebral no tuvo diferencias significativas en el grupo experimental respecto al grupo control; asimismo en cuanto al manejo quirúrgico, entre ambos grupos no se favoreció con el uso de ácido ascórbico.

Es importante aclarar que, debido a la naturaleza del estudio, el uso de fármacos debe ser vigilado de manera estrecha y en el caso de nuestro estudio, el ácido ascórbico mostró bioseguridad, ya que no se tuvieron reacciones adversas durante o posterior a la administración en el grupo experimental.
En cuanto a la mortalidad a los 28 días, Ilama la atención que tras el análisis con dos pruebas estadísticas, análisis de supervivencia con un modelo de regresión de Cox con un HR de 0.128 y análisis por medio de curva de Kaplan-Meier $(p=0.010)$, favorecen el empleo de esta práctica. Con base en este análisis estadístico, podemos sugerir que el uso de ácido ascórbico puede ser un factor protector para mortalidad a los 28 días, coincidiendo con lo publicado por Razmon y su equipo en donde se registró menor mortalidad en el grupo experimental $(p=0.04)$.

En este sentido, la administración de ácido ascórbico en altas dosis mediante vía intravenosa podría disminuir la mortalidad en pacientes con TCE severo, aunque sigue habiendo falta de evidencia sólida para mejoría de estancia hospitalaria y disminución del edema cerebral, por lo que es necesario continuar con ensayos clínicos con mayor número de participantes. Otra de las ventajas del ácido ascórbico es su bajo costo y su alta disponibilidad en los hospitales de segundo y tercer nivel, por lo que creemos que es posible la reproducibilidad de más estudios de esta naturaleza.

Dentro de las limitaciones en este estudio, se encuentra la cantidad de pacientes incluidos, ya que nuestro hospital obedeció a la conversión de áreas destinadas a la actual pandemia (COVID-19), por lo que el número de pacientes disminuyó notablemente impactando en nuestra recolección. Otra debilidad identificada es inherente a la aleatorización de los participantes, en donde se evidenció que los pacientes más graves se encontraron en el grupo experimental, lo que pudo influir en los resultados comentados. Creemos que la búsqueda de estrategias hacia la mejora de este grupo de pacientes con TCE severo debe continuar y que este estudio debe sentar precedentes en nuestro país para la realización de nuevos y mejores estudios que avalen el uso de ésta y otras estrategias que pueden resultar en beneficio comprobable en nuestros pacientes y que formen parte de un bundle de manejo.

\section{CONCLUSIONES}

El uso de ácido ascórbico en el contexto de TCE severo impactó de manera negativa en la estancia en la $\mathrm{UCl}$, así como en el tiempo de hospitalización. No se encontró diferencia en ambos grupos en cuanto a escalas pronósticas funcionales a su egreso, aunque el análisis de supervivencia mostró una menor mortalidad a los 28 días para el grupo experimental respecto a los controles, siendo estadísticamente significativo.

\section{REFERENCIAS}

1. Centers for Disease Control and Prevention. Surveillance report of traumatic brain injury-related emergency department visits, hospitalizations, and deaths. [Accessed November 13, 2019] https://www.cdc.gov/traumaticbraininjury/get_the_facts.html. 
2. Raúl CE, Martín MMJ. Trauma craneoencefálico. Rev Mex de Anestesiología. 2015;38:S433-S4.

3. Guía de Práctica Clínica GPC Atención inicial de pacientes con traumatismo craneoencefálico grave en urgencias. México: Instituto Mexicano del Seguro Social; 2018.

4. Timmons SD. Controversies in severe traumatic brain injury management. Springer, editor. Springer: Springer; 2018. p. 285.

5. Taylor CA, Bell JM, Breiding MJ, Xu LK. Traumatic brain injury-related emergency department visits, hospitalizations, and deaths-United States, 2007 and 2013. MMWR Surveill Summ. 2017;66(9):1-18.

6. Leichtle SW, Sarma AK, Strein M, Yajnik V, Rivet D, Sima A, et al. High-dose intravenous ascorbic acid: ready for prime time in traumatic brain injury? Neurocrit Care. 2020;32(1):333-339.

7. SINAVE. Aspectos clínicos y epidemiológicos del trauma craneoencefálico en México 2008. [Consultada el 22 de diciembre de 2019] https://www.gob.mx/cms/uploads/ attachment/file/13606/2008_sem26.pdf.

8. Teasdale G, Maas A, Lecky F, Manley G, Stocchetti N, Murray G. The Glasgow Coma scale at 40 years: standing the test of time. Lancet Neurol. 2014;13(8):844-54.

9. Munana-Rodríguez JE, Elías AR. Escala de coma de Glasgow: origen, análisis y uso apropiado. Enferm Univ. 2014;11(1):24-35.

10. Carney N, Totten AM, O'Reilly C, Ullman JS, Hawryluk GWJ, Bell $\mathrm{MJ}$, et al. Guidelines for the management of severe traumatic brain injury. 4th ed. Neurosurgery. 2017;80(1):6-15.

11. Picetti E, Rossi S, Abu-Zidan FM, Ansaloni L, Armonda R, Baiocchi GL, et al. WSES consensus conference guidelines: monitoring and management of severe adult traumatic brain injury patients with polytrauma in the first 24 hours. World $J$ Emerg Surg. 2019;14(1):53.

12. Stocchetti N, Carbonara M, Citerio G, Ercole A, Skrifvars MB, Smielewski $P$, et al. Severe traumatic brain injury: targeted management in the intensive care unit. Lancet Neurol. 2017;16(6):452-464.

13. Baratloo A, Shokravi M, Safari S, Aziz AK. Predictive value of Glasgow coma score and full outline of unresponsiveness score on the outcome of multiple trauma patients. Arch Iran Med. 2016;19(3):215-220.

14. Brown AW, Pretz CR, Bell KR, Hammond FM, Arciniegas DB, Bodien YG, et al. Predictive utility of an adapted Marshall head CT classification scheme after traumatic brain injury. Brain Injury. 2019;33(5):610-617.

15. Kinoshita K. Traumatic brain injury: pathophysiology for neurocritical care. J Intensive Care. 2016;4:29.

16. Polidori MC, Mecocci P, Frei B. Plasma vitamin C levels are decreased and correlated with brain damage in patients with intracranial hemorrhage or head trauma. Stroke. 2001;32(4):898-902.

17. Song J, Park J, Kim JH, Choi JY, Kim JY, Lee KM, et al. Dehydroascorbic acid attenuates ischemic brain edema and neurotoxicity in cerebral ischemia: an in vivo study. Exp Neurobiol. 2015;24(1):41-54.

18. Ishaq GM, Saidu Y, Bilbis LS, Muhammad SA, Jinjir N, Shehu BB. Effects of $\alpha$-tocopherol and ascorbic acid in the severity and management of traumatic brain injury in albino rats. $J$ Neurosci Rural Pract. 2013;4(3):292-297.

19. Winkler EA, Minter D, Yue JK, Manley GT. Cerebral edema in traumatic brain injury pathophysiology and prospective therapeutic targets. Neurosurg Clin N Am. 2016;27(4):473-488.
20. Jain KK. The handbook of neuroprotection. 2 ed. Springer Nature: Humana Press; 2019. p. 865.

21. Broderick JP, Adeoye O, Elm J. Evolution of the modified rankin scale and its use in future stroke trials. Stroke. 2017;48(7):2007-2012.

22. McMillan T, Wilson L, Ponsford J, Levin H, Teasdale G, Bond $M$. The Glasgow outcome scale- 40 years of application and refinement. Nat Rev Neurol. 2016;12(8):477-485.

23. Yimcharoen M, Kittikunnathum S, Suknikorn C, Nak-on W, Yeethong $\mathrm{P}$, Anthony TG, et al. Effects of ascorbic acid supplementation on oxidative stress markers in healthy women following a single bout of exercise. J Int Soc Sports Nut. 2019;16(1):2.

24. Fowler AA, Syed AA, Knowlson S, Sculthorpe R, Farthing D, DeWilde $\mathrm{C}$, et al. Phase I safety trial of intravenous ascorbic acid in patients with severe sepsis. J Trans/ Med. 2014;12:32.

25. May JM. Vitamin $\mathrm{C}$ transport and its role in the central nervous system. Sub-cellular Biochem. 2012;56:85-103.

26. Buehner M, Pamplin J, Studer L, Hughes RL, King BT, Graybill $\mathrm{JC}$, et al. Oxalate nephropathy after continuous infusion of highdose vitamin $\mathrm{c}$ as an adjunct to burn resuscitation. J Burn Care Res. 2016;37(4):e374-379.

27. Nathens AB, Neff MJ, Jurkovich GJ, Klotz P, Farver K Ruzinski JT, et al. Randomized, prospective trial of antioxidant supplementation in critically ill surgical patients. Ann Surg. 2002;236(6):814-822.

28. Sanford K, Fisher BJ, Fowler E, Fowler AA, Natarajan R. Attenuation of red blood cell storage lesions with vitamin C. Antioxidants. 2017;6(3):55.

29. Figueroa-Mendez R, Rivas-Arancibia S. Vitamin C in health and disease: its role in the metabolism of cells and redox state in the brain. Front Physiol. 2015;6:397.

30. Kelly PJ, Morrow JD, Ning MM, Koroshetz W, Lo EH, Terry E, et al. Oxidative stress and matrix metalloproteinase- 9 in acute ischemic stroke - The biomarker evaluation for antioxidant therapies in stroke (BEAT-stroke) study. Stroke. 2008;39(1):100104.

31. Razmkon A, Sadidi A, Sherafat-Kazemzadeh E, Mehrafshan A, Jamali M, Malekpour B, et al. Administration of vitamin C and vitamin $\mathrm{E}$ in severe head injury: a randomized double-blind controlled trial. Clin Neurosurg. 2011;58:133-137.

Patrocinios: No se utilizó ningún recurso fuera de los otorgados por la institución médica.

Conflicto de intereses: El autor declara no tener algún conflicto de intereses.

\author{
Correspondencia: \\ Dr. Juan Diego de Jesús Macías Limón \\ Niebla Núm. 323 \\ Col. Jardines del Moral, 37160 , \\ León, Guanajuato, México. \\ Tel.: (477) 7174800 ext. 31725 . \\ E-mail: juandiegodejess@gmail.com
}

